\title{
Intelligence and Brain Efficiency: Investigating the Association between Working Memory Performance, Glutamate, and GABA
}

\author{
Anouk Marsman ${ }^{1 *}$, René C. W. Mandl', Dennis W. J. Klomp², Wiepke Cahn', \\ René S. Kahn', Peter R. Luijten ${ }^{2}$ and Hilleke E. Hulshoff Pol' \\ ${ }^{1}$ Brain Center Rudolf Magnus, Department of Psychiatry, University Medical Center Utrecht, Utrecht, Netherlands, \\ ${ }^{2}$ Department of Radiology, University Medical Center Utrecht, Utrecht, Netherlands
}

OPEN ACCESS

Edited by:

Paul Croarkin,

Mayo Clinic,

United States

Reviewed by:

Ernest Pedapati,

Cincinnati Children's Hospital

Medical Center, United States

Frank P. MacMaster,

University of Calgary, Canada

*Correspondence:

Anouk Marsman

anoukm@drcmr.dk

Specialty section:

This article was submitted to

Neuroimaging and Stimulation,

a section of the journal

Frontiers in Psychiatry

Received: 31 May 2017 Accepted: 07 August 2017 Published: 15 September 2017

Citation:

Marsman A, Mandl RCW, Klomp DWJ, Cahn W, Kahn RS, Luijten PR and Hulshoff Pol HE

(2017) Intelligence and Brain

Efficiency: Investigating the

Association between Working

Memory Performance, Glutamate,

and GABA.

Front. Psychiatry 8:154.

doi: 10.3389/fpsyt.2017.00154
Intelligence is a measure of general cognitive functioning capturing a wide variety of different cognitive functions. It has been hypothesized that the brain works to minimize the resources allocated toward higher cognitive functioning. Thus, for the intelligent brain, it may be that not simply more is better, but rather, more efficient is better. Energy metabolism supports both inhibitory and excitatory neurotransmission processes. Indeed, in glutamatergic and GABAergic neurons, the primary energetic costs are associated with neurotransmission. We tested the hypothesis that minimizing resources through the excitation-inhibition balance encompassing gamma-aminobutyric acid (GABA) and glutamate may be beneficial to general cognitive functioning using $7 \mathrm{~T}^{1} \mathrm{H}-\mathrm{MRS}$ in 23 healthy individuals (male/female $=16 / 7,27.7 \pm 5.3$ years). We find that a higher working memory index is significantly correlated with a lower GABA to glutamate ratio in the frontal cortex and with a lower glutamate level in the occipital cortex. Thus, it seems that working memory performance is associated with the excitation-inhibition balance in the brain.

Keywords: ${ }^{1} \mathrm{H}-\mathrm{MRS}, 7 \mathrm{~T}$, gamma-aminobutyric acid, glutamate, intelligence

\section{INTRODUCTION}

Intelligence is a measure of general cognitive functioning capturing a wide variety of different cognitive functions (1). Intelligence has long been (albeit modestly) associated with brain size $(2-4)$. More recently, intellectual functioning has been implicated in brain functioning (5) and in the efficiency of the functional (6) and structural brain network (7). Regional structural differences in relationship to intelligence have been demonstrated in several studies in healthy individuals $(2,3,8)$ and in individuals with local brain lesions (9). However, it is not known how the human brain handles complex cognitive tasks while being such an expensive organ to operate, utilizing some $20 \%$ of all oxygen taken in and $25 \%$ of all glucose produced while representing only about $2 \%$ of the body's weight $(10,11)$. Indeed, it has been hypothesized that the brain works to minimize the resources allocated toward higher cognitive functioning (12). Thus, for the intelligent brain, it 
may be that not simply more is better, but rather more efficient is better.

Glutamate (Glu) and gamma-aminobutyric acid (GABA) are the major excitatory and inhibitory neurotransmitters in the central nervous system. In both glutamatergic and GABAergic neurons, the primary energetic costs are associated with neurotransmission, and the energetic needs of these neurons dominate the cerebral cortex energy requirements $(13,14)$. In the resting awake state, $80 \%$ of energy used by the brain supports events associated with neuronal firing and cycling of GABA and glutamate, and in the actively awake individual, the change in energy (and its coupled activity) induced by stimulation during task performance is very small in comparison to its baseline value (15). Thus, minimizing resources through the inhibitionexcitation balance encompassing GABA and glutamate may be beneficial to general cognitive functioning. While general intellectual functioning has been related to brain neurochemistry in several studies, measuring largely positive associations with the brain metabolite $N$-acetyl aspartate (NAA) $(16,17)$, a marker of neuronal integrity $(18,19)$, these measures do not provide information on the inhibition-excitation balance. To obtain such information, one needs to reliably measure both glutamate and GABA levels, which is not an easy task using MR scanners operating at conventional magnetic field strengths.

Proton magnetic resonance spectroscopy ( $\left.{ }^{1} \mathrm{H}-\mathrm{MRS}\right)$ at a field strength of $7 \mathrm{~T}$ has an increased sensitivity and spectral resolution. For instance, at $7 \mathrm{~T}$, it is now possible to adequately separate the glutamate and glutamine signals resulting in a higher accuracy of glutamate measurement (20). Despite the increased sensitivity, measurement of GABA is not straightforward because of its low concentration compared to other brain metabolites and its obscured signal due to overlapping signals of higher intensity. To overcome this problem, spectral editing techniques can be applied to isolate the GABA signal (21). Using these methods, we were able to show that higher cognitive functioning was associated with lower GABA levels in the prefrontal cortex in patients with schizophrenia (22).

We explored possible associations between the excitationinhibition balance in the brain and intelligence in the prefrontal cortex and in the occipital cortex. For this purpose, we measured GABA and glutamate levels using ${ }^{1} \mathrm{H}$-MRS at a magnetic field strength of $7 \mathrm{~T}$ in healthy adults. While a whole brain approach would be ideal for studying intelligence, it is currently not feasible to measure GABA and glutamate reliably at a whole brain level using MRS. Therefore, we chose the prefrontal and occipital cortex because the prefrontal cortex has long been found to be important to general cognitive functioning and because the occipital cortex could be considered as a control area. This selection of areas has limitations. Indeed, the frontal lobes are often considered the primary focus of human intelligence $(23,24)$. However, the parieto-frontal integration theory for intelligence (25-27), an association with the whole brain network with intelligence (6), and association of intelligence level with cortical thickness change in many brain areas, including prefrontal and occipital cortices (28) suggest that many different parts of the brain may be involved in intelligence. Moreover, the occipital cortex was also involved in earlier studies [e.g., Ref. (23)]. We hypothesized that the prefrontal cortex is essential for intelligence and thus expect an association with GABA and glutamate and intelligence level in this brain area and not in the occipital cortex in this exploratory study.

\section{MATERIALS AND METHODS}

\section{Subjects}

A total of 23 healthy individuals (16 males/7 females) participated in the study. Participants had no major psychiatric or neurological history, no history of drug or alcohol abuse, and no first-degree relatives with psychiatric or neurological disorders. The study was approved by the Medical Ethics Committee of the University Medical Center Utrecht, The Netherlands, and performed according to the directives of the Declaration of Helsinki (amendment of Seoul, 2008). Participants provided written informed consent prior to the examination. Mean (SD) age was 27.7 (5.3) years, and average completed years of education was $14.1 \pm 2.1$ years.

\section{Cognitive Assessment}

All participants underwent a general cognitive assessment using the full Wechsler Adult Intelligence Scale (WAIS-III-NL) (29). The total intelligence quotient (TIQ) as well as the verbal (VIQ) and performance (PIQ) intelligence quotients, the perceptual reasoning index (PRI), verbal comprehension index (VCI), and the working memory index (WMI) were measured.

\section{MR Acquisition}

All investigations were performed on a $7 \mathrm{~T}$ whole body MR scanner (Philips, Cleveland, OH, USA). A birdcage transmit head coil was used in dual transmit driven by $2 \times 4 \mathrm{~kW}$ amplifiers, in combination with a 32-channel receive coil (both Nova Medical, Inc., Burlington, MA, USA).

For anatomical reference and gray and white matter tissue classification, a $\mathrm{T}_{1}$-weighted magnetization prepared rapid gradient echo sequence was obtained (450 slices, slice thickness $=0.8 \mathrm{~mm}, \mathrm{TR}=7 \mathrm{~ms}, \mathrm{TE}=3 \mathrm{~ms}$, flip angle $=8^{\circ}$, $\mathrm{FOV}=250 \mathrm{~mm} \times 200 \mathrm{~mm} \times 180 \mathrm{~mm}, 312 \times 312$ acquisition matrix, SENSE factor 2.7, scan duration $=408 \mathrm{~s}$ ).

For the assessment of glutamate, ${ }^{1} \mathrm{H}$-MRS experiments were conducted using a sLASER sequence (semi-localized by adiabatic selective refocusing; $\mathrm{TE}=28 \mathrm{~ms}, 32$ averages, $\mathrm{TR}=5 \mathrm{~s}$ ) (Figure 1A). Voxels $(2 \mathrm{~cm} \times 2 \mathrm{~cm} \times 2 \mathrm{~cm})$ were located in the medial prefrontal and medial occipital lobe (Figure 2). Non-water-suppressed spectra were obtained for quantification (carrier frequency was set to the chemical shift of $\mathrm{H}_{2} \mathrm{O}$, acquisition time $=10 \mathrm{~s}$ ).

GABA-edited ${ }^{1} \mathrm{H}$-MRS experiments were conducted using a MEGA-sLASER sequence $(\mathrm{TE}=74 \mathrm{~ms}, 64$ averages, $\mathrm{TR}=4 \mathrm{~s}$ ) (Figure 1B) (31). Voxels $(2.5 \mathrm{~cm} \times 2.5 \mathrm{~cm} \times 2.5 \mathrm{~cm})$ were located in the medial frontal and medial occipital region (Figure 2). Prior to the MRS exams, second order $\mathrm{B}_{0}$ shimming was applied using the FASTERMAP algorithm at the voxel of interest (30, 32). Second, at this location, a high $B_{1}$ field was generated to minimize chemical shift displacement artifacts (33). The 

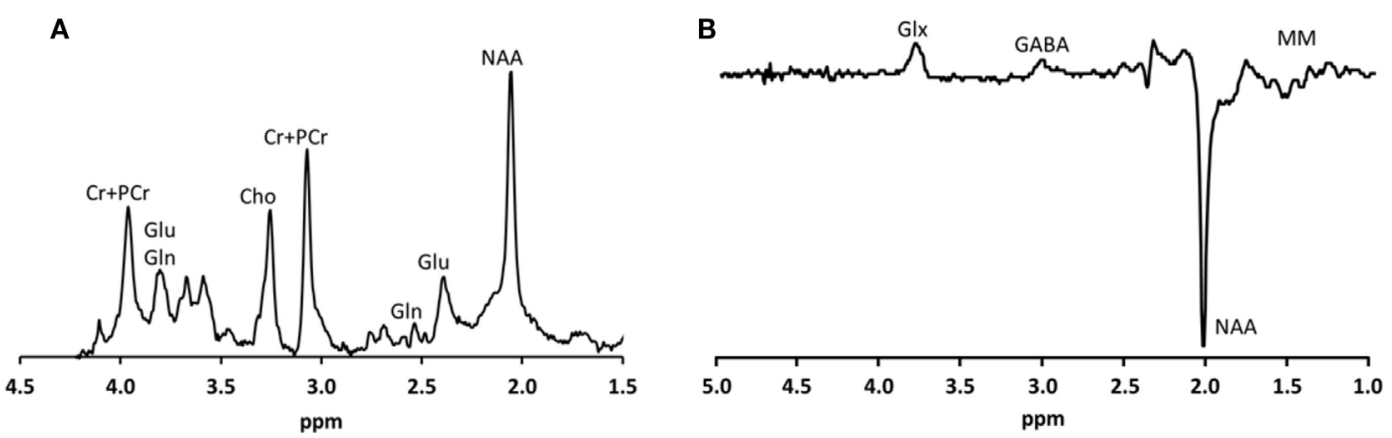

FIGURE 1 | Typical metabolite spectra (A) as recorded using the sLASER sequence and (B) as recorded using the MEGA-sLASER sequence.

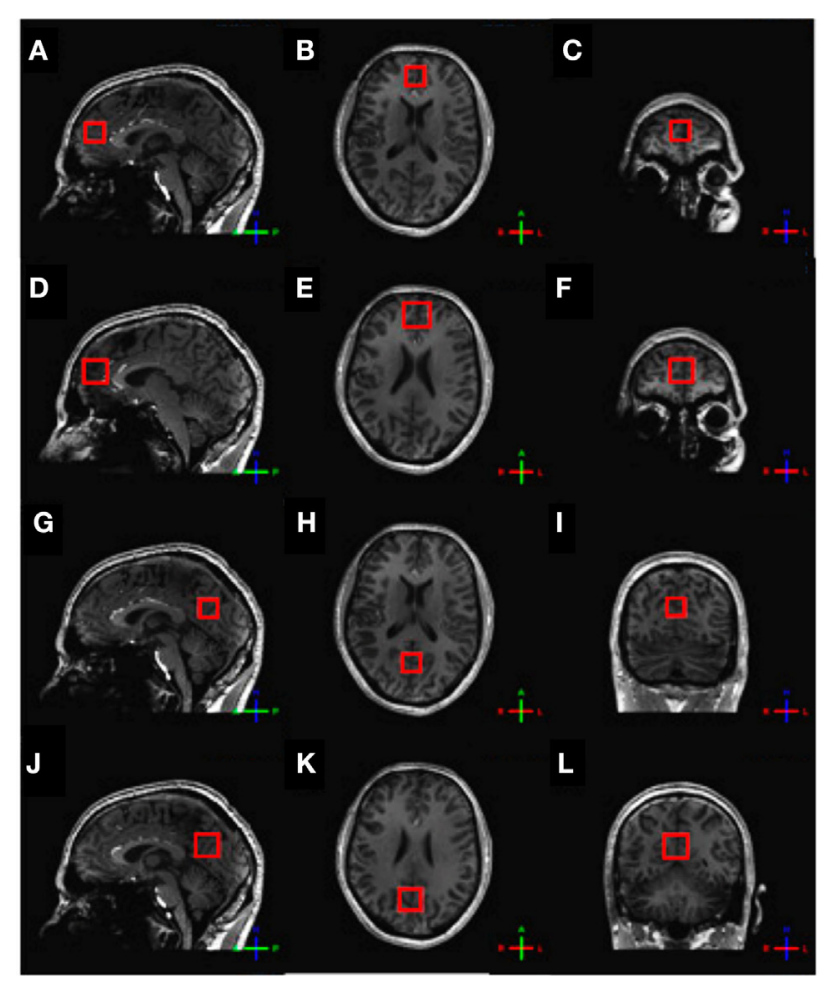

FIGURE 2 | Voxel placement. (A) Frontal sLASER voxel, sagittal view; (B) frontal sLASER voxel, axial view; (C) frontal sLASER voxel, coronal view; (D) frontal MEGA-sLASER voxel, sagittal view; (E) frontal MEGA-sLASER voxel, axial view; (F) frontal MEGA-sLASER voxel, coronal view; (G) occipital sLASER voxel, sagittal view; (H) occipital sLASER voxel, axial view; (I) occipital sLASER voxel, coronal view; (J) occipital MEGA-sLASER voxel, sagittal view; (K) occipital MEGA-sLASER voxel, axial view; (L) occipital MEGA-sLASER voxel, coronal view [from Ref. (22)].

highest possible $\mathrm{B}_{1}$ field was generated by optimizing the phase of both transmit channels to locally assure constructive $B_{1}$ interferences (34).

\section{Spectral Fitting and Quantification}

Retrospective phase and frequency alignment was performed on all data sets of each measurement (35). Fitting of the sLASER spectra was performed with LCModel-based software implemented in Matlab (36), which uses a priori knowledge of the spectral components to fit metabolite resonances (37). The following 16 metabolites and a measured macromolecular baseline (38) were fitted to the spectra: acetate, aspartate, choline (Cho), phosphorylcholine, glycerophosphorylcholine, phosphorylethanolamine, creatine (Cr), phosphocreatine (PCr), NAA, $N$-acetyl aspartyl glutamate, GABA, Glu, glutamine (Gln), glutathione (GSH), myo-inositol (mIns), and taurine (Tau). Glutamate levels were estimated using the water signal as an internal reference and calculated as follows:

$[\mathrm{met}]=$

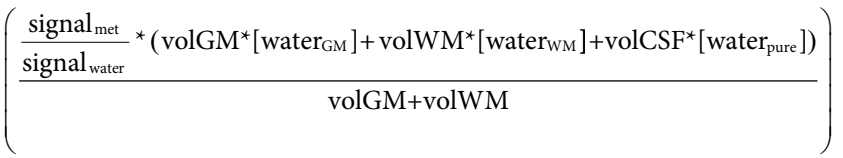

where [met] is the metabolite concentration, signal ${ }_{\text {met }}$ is the fitted signal intensity of the metabolite, accounting for the number of protons, and signal ${ }_{\text {water }}$ is the fitted signal intensity of water, accounting for the number of protons; volGM, volWM, and volCSF are, respectively, the gray matter fraction, white matter fraction, and cerebrospinal fluid (CSF) fraction in the voxel; and

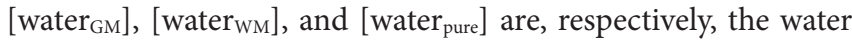
concentration in gray matter, white matter, or CSF. For determining the contribution of gray matter, white matter, and CSF of each voxel, the software package SPM8 was used to segment the $\mathrm{T}_{1}$-weighted image. In the $\mathrm{T}_{1}$-weighted image, the position of the ${ }^{1} \mathrm{H}$-MRS voxel was determined, after which the amount of gray matter, white matter, and CSF in the ${ }^{1} \mathrm{H}-\mathrm{MRS}$ voxel was computed. To account for differences in transverse relaxation between water and metabolites, a correction was applied based on reported $\mathrm{T}_{2}$ values at $7 \mathrm{~T}$ of $47 \mathrm{~ms}$ on average for water and $107 \mathrm{~ms}$ assumed for the metabolites (39). Statistical analysis of the gray and white matter fractions in the frontal and occipital MEGA-sLASER (GABA/Cr) and sLASER (glutamate) revealed correlations $>0.95$ for both gray and white matter fractions in the two voxels.

Fitting of the MEGA-sLASER spectra was performed by frequency-domain fitting of the GABA and creatine resonances to a Lorentzian line-shape function in Matlab. GABA levels were expressed as the ratios of their peak areas relative to the peak areas of the creatine resonance (GABA/Cr). 


\section{Statistical Analysis}

Statistical analyses were performed using SPSS 21.0 (2012, Chicago, IL, USA). Data were controlled for their normality of the distributions. No transformations for correction were needed. To evaluate differences in metabolite concentrations and gray and white matter fractions between the frontal and occipital areas, paired $t$-tests were done. To evaluate associations between brain metabolite levels with general intelligence measures, partial correlation coefficients were done with corrections for age, sex, and for gray and white matter fractions (40). The partial correlation between IQ and metabolite levels was defined as the correlation between the residuals of IQ and metabolite levels resulting from the linear regressions of IQ and metabolite levels with the controlling variables, i.e., age, sex, and gray and white matter fractions of the sLASER and/or MEGA-sLASER voxels. To correct for multiple comparisons, while taking the considerable dependency among intelligence quotient measures and the exploratory nature of the study into account, we restricted the significance level of the correlations to $p \leq 0.01$.

\section{RESULTS}

\section{Intelligence Measures}

The mean (SD) of the TIQ was 108 (13), the verbal intelligence quotient (VIQ) was 109 (12), the performance intelligence quotient (PIQ) was 107 (14), the VCI was 111 (13), the PRI was 108 (15), and the WMI was 105 (12) (Table 1).

\section{Metabolite Concentrations in the Frontal and Occipital Areas}

Because of poor spectral quality as established by a Cramér-Rao lower bound of more than $20 \%$ and visual inspection, some data were excluded from the study. Frontal MRS results are based on 18 subjects and occipital MRS results are based on 17 subjects. Frontal GABA-edited MRS results are based on 19 subjects and occipital GABA-edited MRS results are also based on 19 subjects.

Paired $t$-tests for differences in metabolite concentrations and gray and white matter fractions between the frontal and occipital areas revealed no significant differences except for a higher white matter fraction in the occipital sLASER voxel, but this finding did not survive the analysis after correction for age and sex (Table 1).

\section{Brain Metabolites, Gray and White Matter Fractions, and Intelligence}

A higher WMI was significantly correlated with a lower GABA/ Glu ratio (GABA/Cr to $\mathrm{Glu} / \mathrm{Cr}$ ratio) in the frontal cortex $[r(7)=-0.80, p=0.01]$ and not significantly in the occipital cortex $[r(7)=0.68, p=0.04]$ (Table 2; Figure 3).

A higher WMI was not significantly correlated with the frontal GABA/Cr ratio $[r(10)=-0.05, p=n s]$, frontal glutamate concentration $[r(10)=-0.53, p=0.076]$, and occipital GABA/ Cr ratio $[r(9)=0.42, p=0.19]$. A higher WMI was significantly associated with a lower occipital glutamate concentration $[r(10)=-0.79, p<0.004]$ (Table 2; Figure 4).

No significant associations were found for TIQ, Verbal intelligence quotient, performance intelligence quotient, VCI, and PRI with any of the metabolites in the frontal and occipital cortices. All correlations with metabolites were corrected for age, sex, and gray and white matter fractions.

\section{Brain Metabolites, Age, and Sex}

There were no other significant associations between metabolite levels in the prefrontal and occipital cortices with age. These correlations were corrected for sex, and gray and white matter fractions. There were no significant associations between metabolite levels in the prefrontal and occipital cortices with sex.

\section{Correlations among Metabolites}

Following correction for age, sex, gray and white matter fractions, in the occipital cortex, a significant negative correlation

TABLE 1 | Intelligence and brain metabolites in healthy individuals. ${ }^{a}$

\begin{tabular}{|c|c|c|c|c|}
\hline Intelligence & (Sub) test score & Mean (SD) & Min & Max \\
\hline & Total IQ & $108(13)$ & 82 & 131 \\
\hline & Verbal IQ & 109 (12) & 82 & 128 \\
\hline & Performance IQ & $107(14)$ & 78 & 127 \\
\hline & Verbal comprehension index & $111(13)$ & 91 & 132 \\
\hline & Perceptual reasoning index & $108(15)$ & 79 & 129 \\
\hline & Working memory index & $105(12)$ & 86 & 124 \\
\hline${ }^{1} \mathrm{H}-\mathrm{MRS}$ sequence & Metabolite & Prefrontal [mean (SD)] & Occipital [mean (SD)] & $\begin{array}{c}\text { Paired } \\
\text { t-test }\end{array}$ \\
\hline \multirow[t]{3}{*}{ MEGA-SLASER } & GABA/Cr ratio & $0.14(0.03)$ & $0.13(0.03)$ & ns \\
\hline & Gray matter (\%) & $68.1(11.3)$ & $68.0(11.9)$ & ns \\
\hline & White matter (\%) & $24.0(11.8)$ & $27.7(13.2)$ & ns \\
\hline \multirow[t]{3}{*}{ SLASER } & Glutamate (mM) & $8.65(1.14)$ & $8.48(1.26)$ & ns \\
\hline & Gray matter (\%) & $71.4(14.3)$ & $70.1(12.7)$ & ns \\
\hline & White matter (\%) & $19.5(15.6)$ & $25.2(14.6)$ & $p<0.05^{b}$ \\
\hline
\end{tabular}

ancorrected data based on two separate ${ }^{1} \mathrm{H}-\mathrm{MRS}$ measurements and $T_{1}$-weighted volume measurements performed in two brain areas (medial prefrontal and medial occipital). For assessment of GABA/Cr ratios, a MEGA-sLASER sequence was performed and successfully completed in 19 individuals in the medial prefrontal cortex and in 18 individuals in the medial occipital cortex. A sLASER sequence was performed for assessment of glutamate and successfully completed in 18 individuals in the medial prefrontal cortex and in 17 individuals in the medial occipital cortex. Volume data are based on 21 individuals except for the occipital MEGA-sLASER voxel volumes, which are based on 18 individuals.

${ }^{b}$ Following corrections for age and sex, this difference was no longer significant. 
was found between $\mathrm{GABA} / \mathrm{Cr}$ ratio and glutamate concentration $[r(7)=-0.85, p<0.01]$.

Higher GABA/Cr ratios in the prefrontal cortex were significantly correlated with lower glutamate concentrations $[r(7)=-0.89, p<0.01]$ in the occipital cortex (Figure 5).

\section{DISCUSSION}

To our knowledge, this study presents the first proton magnetic resonance spectroscopy measurements of GABA and glutamate levels in vivo at a magnetic field strength of $7 \mathrm{~T}$ associated with level of intelligence. The main finding is that a higher WMI is associated with a significantly lower GABA/Glu ratio in the

TABLE 2 | Brain metabolites and intelligence. ${ }^{\text {a }}$

\begin{tabular}{lrrrrrr}
\hline Metabolites & \multicolumn{7}{c}{ Cognition } \\
\cline { 2 - 7 } & TIQ & VIQ & PIQ & VCI & PRI & WMI \\
\hline Prefrontal & & & & & & \\
GABA/Cr & 0.31 & 0.28 & 0.30 & 0.25 & 0.37 & -0.05 \\
Glutamate & -0.01 & -0.25 & 0.26 & 0.01 & 0.28 & -0.53 \\
GABA/Glu & -0.51 & -0.67 & -0.19 & -0.41 & -0.13 & $-\mathbf{0 . 8 0 ^ { * * }}$ \\
Occipital & & & & & & \\
GABA/Cr & 0.03 & 0.09 & -0.08 & 0.04 & -0.18 & 0.42 \\
Glutamate & -0.19 & -0.27 & -0.07 & -0.17 & -0.10 & $-\mathbf{0 . 7 9}$ \\
GABA/Glu & 0.14 & 0.22 & -0.06 & 0.05 & -0.19 & $\mathbf{0 . 6 8}^{\star}$ \\
\hline
\end{tabular}

Bold font indicates significant correlations.

aPearson correlations, corrected for age, sex, and for local gray matter and white matter fractions.

${ }^{* *} p \leq 0.01$.

${ }^{*} p \leq 0.05$.

$T I Q$, total intelligence quotient; VIQ, verbal intelligence quotient; $P I Q$, performance intelligence quotient; $\mathrm{VCl}$, verbal comprehension index; PRI, perceptual reasoning index; WMI, working memory index. frontal cortex. However, in contrast to what was expected, we also find a significantly lower glutamate concentration in the occipital cortex. This may suggest that the excitation-inhibition balance in the frontal cortex, and perhaps to a lesser extent in the occipital cortex, is associated with working memory. Working memory refers to the ability to actively hold information on-line over brief periods of time (41). Working memory is one of the factors marking intelligence and has been closely related to general intelligence, although the extent of overlap is point of discussion (42). Moreover, working memory has been positively associated with gray and white matter volume, and with white matter tracts, and these associations are under genetic control $(3,43)$. Interestingly, based on studies in animals, it has been found that a successful working memory performance requires an exquisite balance of the excitatory and inhibitory circuitry in the prefrontal cortex that includes glutamate and GABA (41). Neurons in the prefrontal cortex have been shown to fire persistently during the maintenance phase of working memory tasks for which a balance between inhibitory and excitatory neurons are thought to be required. Supportive evidence for such a system during activation in the human brain was found in a study where a lower resting-state GABA level was associated with higher amplitude of the BOLD fMRI response to a simple visual stimulus in the visual cortex (44) [for review, see Ref. (45)]. Other negative associations were found between resting-state GABA level with visual orientation discrimination performance in the occipital cortex (46), and in the supplementary motor area with tactile discrimination performance $(47,48)$, which also support associations between GABA levels in the human cortex with cognitive functioning. Here, we show, by using ${ }^{1} \mathrm{H}-\mathrm{MRS}$ at $7 \mathrm{~T}$, that lower frontal resting-state GABA/Glu ratios and lower occipital glutamate concentrations may lead to a higher working memory performance in healthy adults, possibly through a more efficient inhibition-excitation balance.
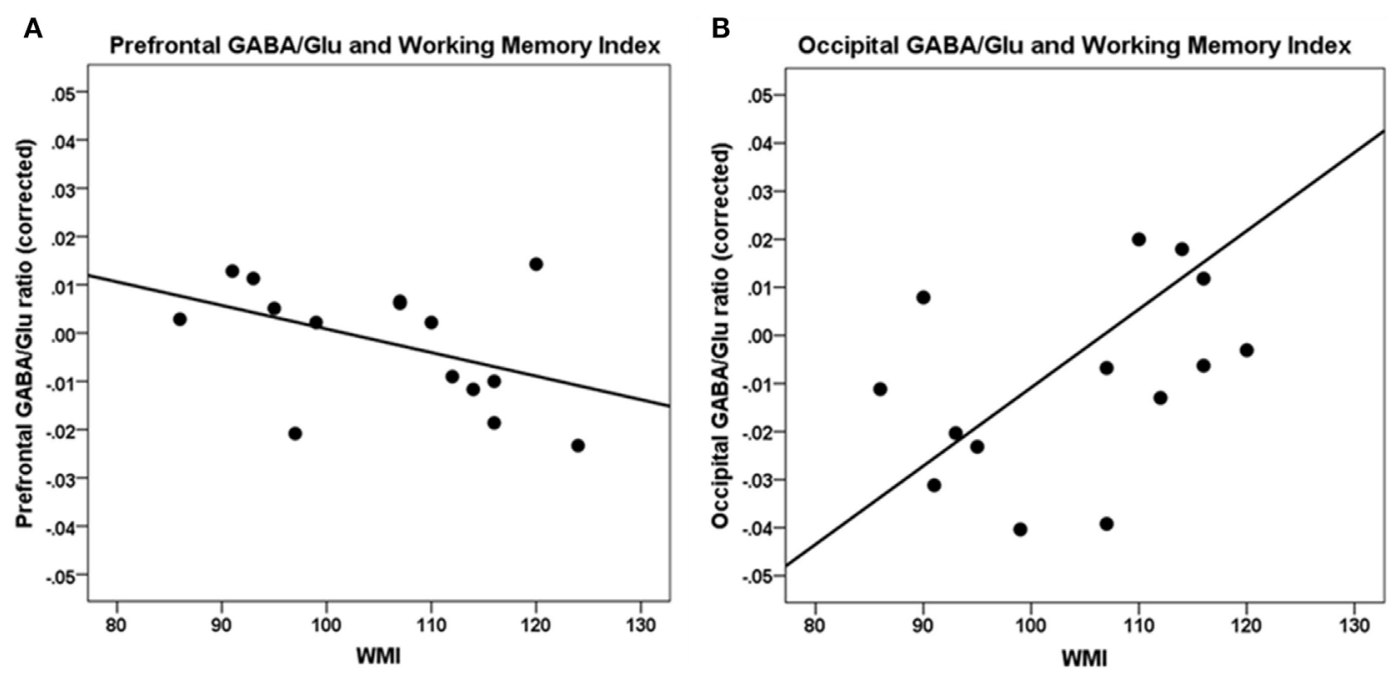

FIGURE 3 | Correlations between working memory index (WMI) and GABA/Glu ratio. (A) Prefrontal GABA/Glu ratios are significantly correlated with WMI $[r(7)=-0.80, p=0.01]$. (B) Occipital GABA/Glu ratios are not significantly correlated with WMI. Data are presented corrected for gray and white matter fractions, age, and sex. 


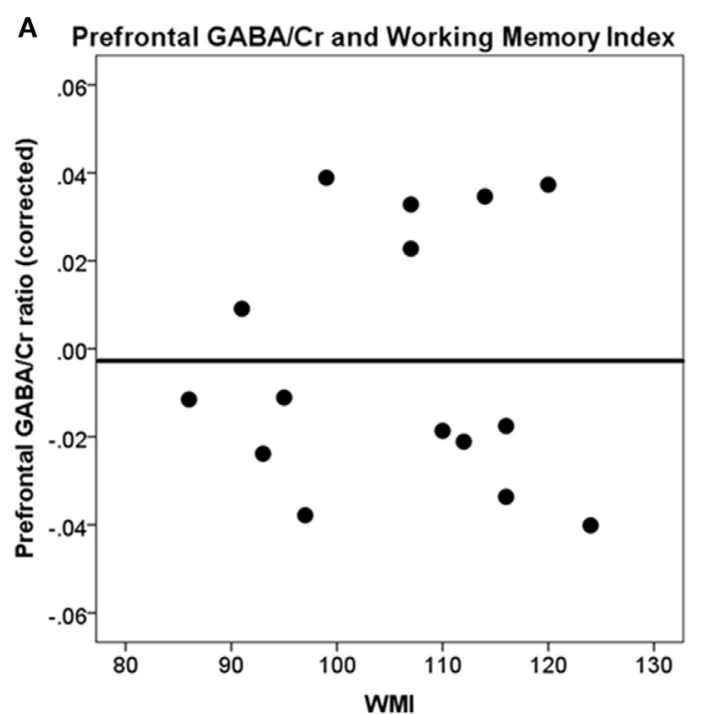

C

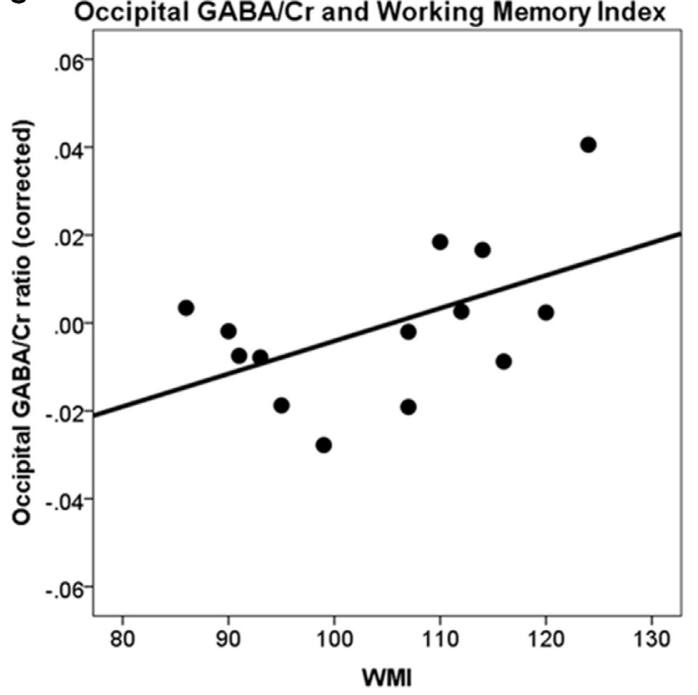

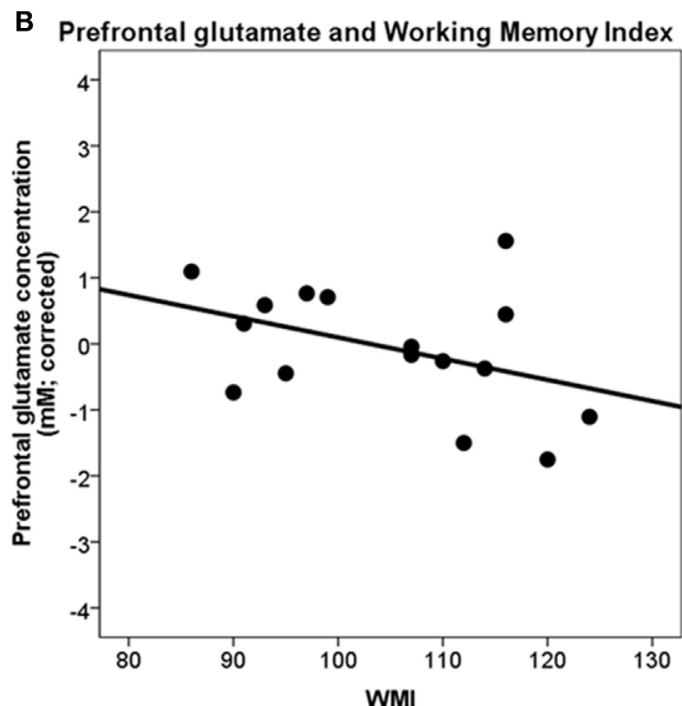

D

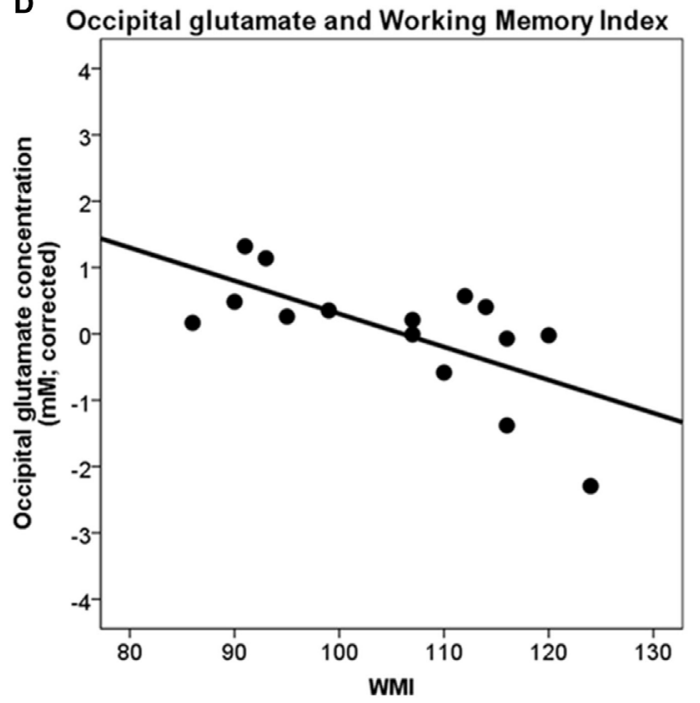

FIGURE 4 | Correlations between prefrontal and occipital GABA/Cr and glutamate with working memory index (WMI). (A) Prefrontal GABA/Cr ratios are not significantly correlated with WMI; (B) prefrontal glutamate concentrations are not significantly correlated with WMI; (C) occipital GABA/Cr ratios are not significantly correlated with WMI; (D) occipital glutamate concentrations are significantly correlated with WMI $[r(10)=-0.79, p<0.004]$. Data are presented corrected for gray and white matter fractions, age, and sex.

Our measurements of brain metabolites were done in the frontal and occipital cortices using SLASER and MEGA-sLASER at $7 \mathrm{~T}$. We could thus measure to which extend the metabolites were correlated within individuals. The most prominent association was found between levels of glutamate and GABA/Cr ratio in the occipital cortex, with higher levels of glutamate being associated with lower levels of GABA/Cr (-0.85). Interestingly, a significant negative correlation between GABA/Cr in the occipital cortex and glutamate level in the prefrontal cortex was also found, thus suggesting a possible differential and maybe connected resting state association between these two anatomically distant brain regions.

This study has some limitations to take into account. One, with MRS, one cannot distinguish intracellular and extracellular metabolite levels. Two, because of its low concentration, a large voxel size is needed to reliably and time-efficiently measure GABA. Hence, the voxel contained both gray and white matter. Three, the stringent correction for multiple comparisons was not possible due to the relatively limited number of participants that were included in the study. We chose for a more lenient approach to allow for the exploration of the association between these metabolite levels and intellectual functioning. Future studies with larger number of participants may allow for a more stringent correction for the multiple comparisons to confirm the stability of these findings.

Future studies using instance modern network analyses (49) may reveal how such connections between metabolite levels act. Such studies may reveal to which extent the associations between 

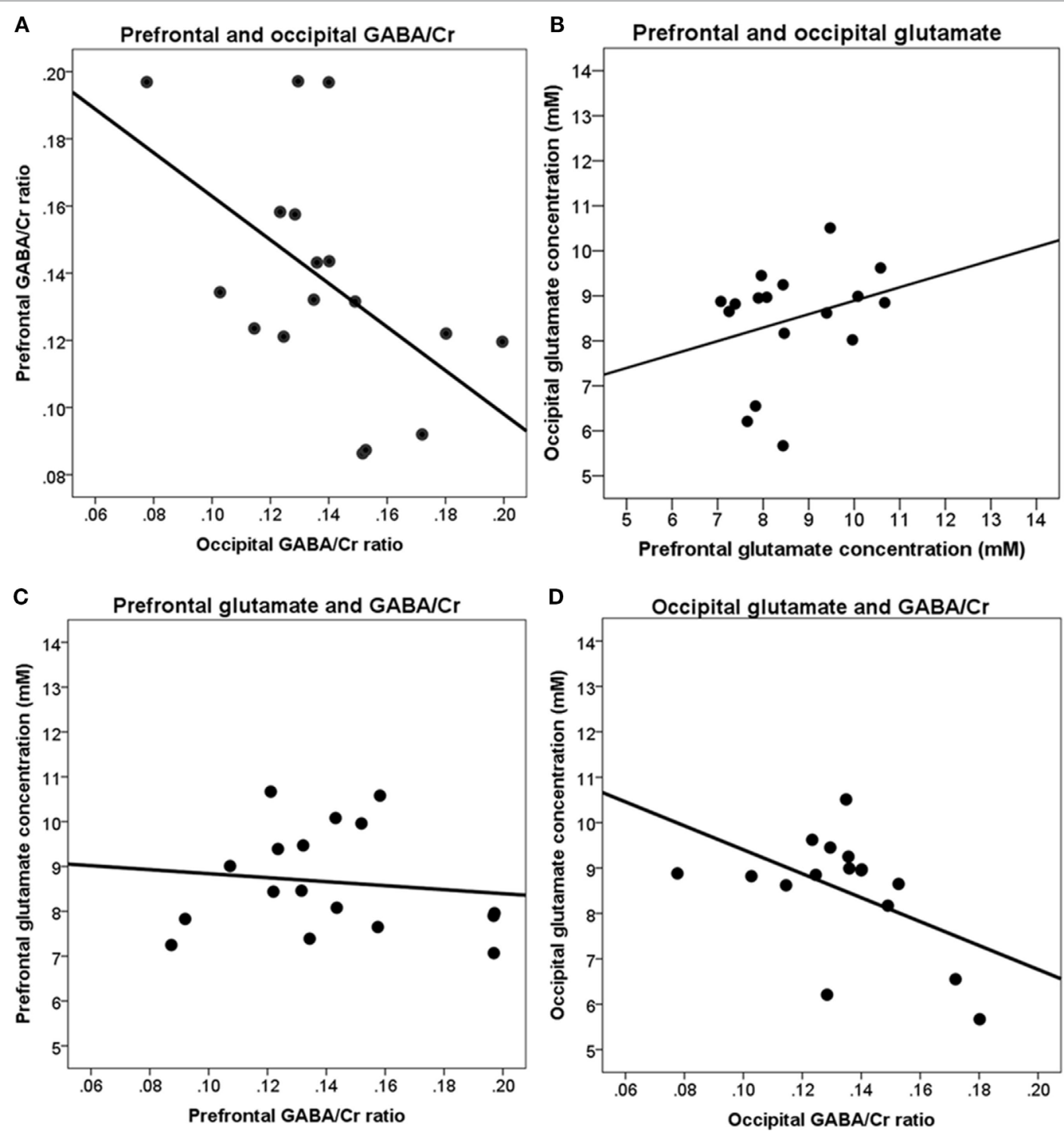

FIGURE 5 | Correlations between brain metabolites in the prefrontal and occipital cortices. (A) Prefrontal and occipital GABA/Cr ratios are not significantly correlated; (B) prefrontal and occipital glutamate concentrations are not significantly correlated; (C) prefrontal GABA/Cr ratios and glutamate concentrations are not significantly correlated; (D) occipital GABA/Cr ratios and glutamate concentrations are significantly correlated $[r(7)=-0.85, p<0.01]$. Data are presented uncorrected for gray and white matter fractions, age, and sex.

intelligence and brain metabolites are linked to local glucose levels and BOLD fMRI effects as well as to network efficiency of these nodes with the rest of the brain. There is evidence that the metabolic costs of a brain area (i.e., a node in a network) are proportional to the number of (mathematical) paths it has to connect with other nodes, and that the metabolic costs of a path are proportional to the physical distance it spans between nodes (50). Interestingly, Brodmann areas with a high glycolytic index were also found to be hub areas (i.e., have a high centrality rank); including Brodmann areas 32 and 33 (51), which are anatomically overlapping with the medial prefrontal voxel location of our current study. A functional neural network study revealed that a higher intelligence was associated with more efficient brain network (6), and thus possibly with a more efficient use of local brain metabolism including GABA and glutamate. Indeed, associations between GABA, glutamate, BOLD signal $(52,53)$, and functional connectivity (54) have been reported. A recent study found that the frontal GABA/Glx ratio (Glx is the sum of the glutamate and glutamine signals) is related to oscillatory modulations during a working memory task, showing that a low GABA/ Glx ratio is needed for efficient inhibition of irrelevant neural activity in precise task performance (55). Changes in GABA and glutamate levels have been found in patients with schizophrenia, a disorder, which is known to affect cognitive functioning $(22,30$, 56). Recent evidence supports the involvement of glutamate and GABA in working memory performance in schizophrenia (57). 
In conclusion, we found that working memory performance is associated with the excitation-inhibition balance in the brain.

\section{ETHICS STATEMENT}

This study was carried out in accordance with the recommendations of the institutional ethics board (METC) with written informed consent from all subjects. All subjects gave written informed consent in accordance with the Declaration of Helsinki. The protocol was approved by the institutional ethics board (METC).

\section{REFERENCES}

1. Deary IJ. Intelligence. Annu Rev Psychol (2012) 63:453-82. doi:10.1146/ annurev-psych-120710-100353

2. Thompson PM, Cannon TD, Narr KL, van ET, Poutanen VP, Huttunen M, et al. Genetic influences on brain structure. Nat Neurosci (2001) 4:1253-8. doi:10.1038/nn758

3. Posthuma D, de Geus EJ, Baare WF, Hulshoff Pol HE, Kahn RS, Boomsma DI. The association between brain volume and intelligence is of genetic origin. Nat Neurosci (2002) 5:83-4. doi:10.1038/nn0202-83

4. Toga AW, Thompson PM. Genetics of brain structure and intelligence. Annu Rev Neurosci (2005) 28:1-23. doi:10.1146/annurev.neuro.28.061604. 135655

5. Song M, Zhou Y, Li J, Liu Y, Tian L, Yu C, et al. Brain spontaneous functional connectivity and intelligence. Neuroimage (2008) 41:1168-76. doi:10.1016/j. neuroimage.2008.02.036

6. van den Heuvel MP, Stam CJ, Kahn RS, Hulshoff Pol HE. Efficiency of functional brain networks and intellectual performance. J Neurosci (2009) 29:7619-24. doi:10.1523/JNEUROSCI.1443-09.2009

7. Chiang MC, Barysheva M, Shattuck DW, Lee AD, Madsen SK, Avedissian C, et al. Genetics of brain fiber architecture and intellectual performance. J Neurosci (2009) 29:2212-24. doi:10.1523/JNEUROSCI.418408.2009

8. Hulshoff Pol HE, Schnack HG, Posthuma D, Mandl RC, Baaré WFC, Van Oel CJ, et al. Genetic contributions to human brain morphology and intelligence. J Neurosci (2006) 26:10235-42. doi:10.1523/JNEUROSCI. 1312-06.2006

9. Glascher J, Tranel D, Paul LK, Rudrauf D, Rorden C, Hornaday A, et al. Lesion mapping of cognitive abilities linked to intelligence. Neuron (2009) 61:681-91. doi:10.1016/j.neuron.2009.01.026

10. Clarke DD, Sokoloff L. Circulation and energy metabolism of the brain. In: Siegel GJ, Agranoff BW, Albers RW, Fisher SK, Uhler MD, editors. Basic Neurochemistry: Molecular, Cellular and Medical Aspects. Philadelphia: Lippincott-Raven (1999). p. 637-70.

11. Raichle ME, Gusnard DA. Appraising the brain's energy budget. Proc Natl Acad Sci U S A (2002) 99:10237-9. doi:10.1073/pnas.172399499

12. Jung RE, Haier RJ. The Parieto-Frontal Integration Theory (P-FIT) of intelligence: converging neuroimaging evidence. Behav Brain Sci (2007) 30:135-54. doi:10.1017/S0140525X07001185

13. Patel AB, De Graaf RA, Mason GF, Rothman DL, Shulman RG, Behar KL. The contribution of GABA to glutamate/glutamine cycling and energy metabolism in the rat cortex in vivo. Proc Natl Acad Sci U S A (2005) 102:5588-93. doi:10.1073/pnas.0501703102

14. Rothman DL, Behar KL, Hyder F, Shulman RG. In vivo NMR studies of the glutamate neurotransmitter flux and neuroenergetics: implications for brain function. Annu Rev Physiol (2003) 65:401-27. doi:10.1146/annurev. physiol.65.092101.142131

15. Shulman RG, Rothman DL, Behar KL, Hyder F. Energetic basis of brain activity: implications for neuroimaging. Trends Neurosci (2004) 27:489-95. doi:10.1016/j.tins.2004.06.005

\section{AUTHOR CONTRIBUTIONS}

AM was involved in the design of the study, data acquisition, data analysis, and writing of the manuscript. $\mathrm{RM}$ and $\mathrm{HH}$ were involved in the design of the study, data analysis, and writing of the manuscript. DK, WC, RK, and PL were involved in the design of the study and revising the manuscript.

\section{ACKNOWLEDGMENTS}

This research was funded by the Netherlands Organisation for Scientific Research (NWO) VIDI Grant 917-46-370 (to HP); Utrecht University High Potential Grant (to HP).

16. Jung RE, Gasparovic C, Chavez RS, Caprihan A, Barrow R, Yeo RA. Imaging intelligence with proton magnetic resonance spectroscopy. Intelligence (2009) 37:192-8. doi:10.1016/j.intell.2008.10.009

17. Ross AJ, Sachdev PS. Magnetic resonance spectroscopy in cognitive research. BrainRes BrainResRev(2004)44:83-102.doi:10.1016/j.brainresrev.2003.11.001

18. Barker PB. N-acetyl aspartate - a neuronal marker? Ann Neurol (2001) 49:423-4. doi:10.1002/ana.90

19. Moffett JR, Ross B, Arun P, Madhavarao CN, Namboodiri AM. $\mathrm{N}$-acetylaspartate in the CNS: from neurodiagnostics to neurobiology. Prog Neurobiol (2007) 81:89-131. doi:10.1016/j.pneurobio.2006.12.003

20. Tkac I, Andersen P, Adriany G, Merkle H, Ugurbil K, Gruetter R. In vivo $1 \mathrm{H}$ NMR spectroscopy of the human brain at 7T. Magn Reson Med (2001) 46:451-6. doi:10.1002/mrm.1213

21. Puts NA, Edden RA. In vivo magnetic resonance spectroscopy of GABA: a methodological review. Prog Nucl Magn Reson Spectrosc (2012) 60:29-41. doi:10.1016/j.pnmrs.2011.06.001

22. Marsman A, Mandl RCW, Klomp DWJ, Bohlken MM, Boer VO, Andreychenko A, et al. GABA and glutamate in schizophrenia: a 7T 1H-MRS study. Neuroimage Clin (2014) 6:398-407. doi:10.1016/j.nicl.2014.10.005

23. Duncan J, Seitz RJ, Kolodny J, Bor D, Herzog H, Ahmed A, et al. A neural basis for general intelligence. Science (2000) 289:457-60. doi:10.1126/ science.289.5478.457

24. Gray JR, Chabris CF, Braver TS. Neural mechanisms of general fluid intelligence. Nat Neurosci (2003) 6(3):316-22. doi:10.1038/nn1014

25. Vakhtin AA, Ryman SG, Flores RA, Jung RE. Functional brain networks contributing to the parieto-frontal integration theory of Intelligence. Neuroimage (2014) 103:349-54. doi:10.1016/j.neuroimage.2014.09.055

26. Haier RJ, Siegel BV, Nuechterlein KH, Hazlett E, Wu JC, Paek J, et al Cortical glucose metabolic rate correlates of abstract reasoning and attention studied with positron emission tomography. Intelligence (1988) 12:199-217. doi:10.1016/0160-2896(88)90016-5

27. Barbey AK, Colom R, Solomon J, Krueger F, Forbes C, Grafman J. An integrative architecture for general intelligence and executive function revealed by lesion mapping. Brain (2012) 135:1154-64. doi:10.1093/brain/ aws 021

28. Schnack HG, van Haren NE, Brouwer RM, Evans A, Durston S, Boomsma DI, et al. Changes in thickness and surface area of the human cortex and their relationship with intelligence. Cereb Cortex (2015) 25(6):1608-17. doi:10.1093/cercor/bht357

29. Wechsler D. Wechsler Adult Intelligence Scale (WAIS-III). Dutch Version. Lisse, The Netherlands: Swets \& Zeitlinger (1997).

30. Gruetter R, Boesch C. Fast, non-iterative shimming on spatially localized signals: in vivo analysis of the magnetic field along axes. J Magn Reson (1992) 96:323-34.

31. Andreychenko A, Boer VO, Arteaga de Castro CS, Luijten PR, Klomp DW. Efficient spectral editing at 7 T: GABA detection with MEGA-sLASER. Magn Reson Med (2012) 68(4):1018-25. doi:10.1002/mrm.24131

32. Gruetter R. Automatic, localized in vivo adjustment of all first- and secondorder shim coils. Magn Reson Med (1993) 29:804-11. doi:10.1002/mrm. 1910290613 
33. Versluis MJ, Kan HE, van Buchem MA, Webb AG. Improved signal to noise in proton spectroscopy of the human calf muscle at $7 \mathrm{~T}$ using localized B1 calibration. Magn Reson Med (2010) 63:207-11. doi:10.1002/mrm. 22195

34. Boer VO, van Lier AL, Hoogduin JM, Wijnen JP, Luijten PR, Klomp DW. 7-T (1) H MRS with adiabatic refocusing at short TE using radiofrequency focusing with a dual-channel volume transmit coil. NMR Biomed (2011) 24:1038-46. doi:10.1002/nbm.1641

35. Waddell KW, Avison MJ, Joers JM, Gore JC. A practical guide to robust detection of GABA in human brain by J-difference spectroscopy at $3 \mathrm{~T}$ using a standard volume coil. Magn Reson Imaging (2007) 25:1032-8. doi:10.1016/j. mri.2006.11.026

36. De Graaf RA. NMR Processing Software for Spectroscopy, Imaging and Spectroscopic Imaging (1999) [Computer Software].

37. Govindaraju V, Young K, Maudsley AA. Proton NMR chemical shifts and coupling constants for brain metabolites. NMR Biomed (2000) 13:129-53. doi:10.1002/1099-1492(200005)13:3<129::AID-NBM619>3.3.CO;2-M

38. Behar KL, Rothman DL, Spencer DD, Petroff OA. Analysis of macromolecule resonances in 1H NMR spectra of human brain. Magn Reson Med (1994) 32:294-302. doi:10.1002/mrm.1910320304

39. Marjanska M, Auerbach EJ, Valabregue R, Van de Moortele PF, Adriany G, Garwood M. Localized 1H NMR spectroscopy in different regions of human brain in vivo at $7 \mathrm{~T}$ : T2 relaxation times and concentrations of cerebral metabolites. NMR Biomed (2012) 25:332-9. doi:10.1002/nbm.1754

40. Morrison DF. Multivariate Statistical Methods. New York: McGraw-Hill (1976).

41. Goldman-Rakic PS. Cellular basis of working memory. Neuron (1995) 14:477-85. doi:10.1016/0896-6273(95)90304-6

42. Ackerman PL, Beier ME, Boyle MO. Working memory and intelligence: the same or different constructs? Psychol Bull (2005) 131:30-60. doi:10.1037/0033-2909.131.1.30

43. Karlsgodt KH, Kochunov P, Winkler AM, Laird AR, Almasy L, Duggirala R, et al. A multimodal assessment of the genetic control over working memory. J Neurosci (2010) 30:8197-202. doi:10.1523/JNEUROSCI.035910.2010

44. Muthukumaraswamy SD, Evans CJ, Edden RA, Wise RG, Singh KD. Individual variability in the shape and amplitude of the BOLD-HRF correlates with endogenous GABAergic inhibition. Hum Brain Mapp (2012) 33:455-65. doi:10.1002/hbm.21223

45. Karlsgodt KH, Bachman P, Winkler AM, Bearden CE, Glahn DC. Genetic influence on the working memory circuitry: behavior, structure, function and extensions to illness. Behav Brain Res (2011) 225:610-22. doi:10.1016/j. bbr.2011.08.016

46. Edden RA, Muthukumaraswamy SD, Freeman TC, Singh KD. Orientation discrimination performance is predicted by GABA concentration and gamma oscillation frequency in human primary visual cortex. JNeurosci (2009) 29:15721-6. doi:10.1523/JNEUROSCI.4426-09.2009

47. Boy F, Evans CJ, Edden RA, Singh KD, Husain M, Sumner P. Individual differences in subconscious motor control predicted by GABA concentration in SMA. Curr Biol (2010) 20:1779-85. doi:10.1016/j.cub.2010. 09.003

48. Puts NA, Edden RA, Evans CJ, McGlone F, McGonigle DJ. Regionally specific human GABA concentration correlates with tactile discrimination thresholds. J Neurosci (2011) 31:16556-60. doi:10.1523/JNEUROSCI.448911.2011

49. Sporns O. Networks of the Brain. Cambridge: The MIT Press (2011).

50. Bullmore E, Sporns O. The economy of brain network organization. Nat Rev Neurosci (2012) 13:336-49. doi:10.1038/nrn3214

51. Vaishnavi SN, Vlassenko AG, Rundle MM, Snyder AZ, Mintun MA, Raichle ME. Regional aerobic glycolysis in the human brain. Proc Natl Acad Sci U S A (2010) 107:17757-62. doi:10.1073/pnas.1010459107

52. Northoff G, Walter M, Schulte RF, BEck J, Dydak U, Henning A, et al. GABA concentrations in the human anterior cingulate cortex predict negative BOLD responses in fMRI. Nat Neurosci (2007) 10:1515-7. doi:10.1038/nn2001

53. Enzi B, Duncan NW, Kaufmann J, Tempelmann C, Wiebking C, Northoff G. Glutamate modulates resting state activity in the perigenual anterior cingulate cortex - a combined fMRI-MRS study. Neuroscience (2012) 227:102-9. doi:10.1016/j.neuroscience.2012.09.039

54. Kapogiannis D, Reiter DA, Willette AA, Mattson MP. Posteromedial cortex glutamate and GABA predict intrinsic functional connectivity of the default mode network. Neuroimage (2013) 64:112-9. doi:10.1016/j. neuroimage.2012.09.029

55. Takei Y, Fujihara K, Tagawa M, Hironaga N, Near J, Kasagi M, et al. The inhibition/excitation ratio related to task-induced oscillatory modulations during a working memory task: a multimodal-imaging study using MEG and MRS. Neuroimage (2016) 128:302-15. doi:10.1016/j.neuroimage.2015. 12.057

56. Marsman A, Boer VO, Luijten PR, Hulshoff Pol HE, Klomp DWJ, Mandl RCW. Detection of glutamate alterations in the human brain using ${ }^{1} \mathrm{H}-\mathrm{MRS}$ : comparison of STEAM and sLASER at 7T. Front Psychiatry (2017) 8:60. doi:10.3389/fpsyt.2017.00060

57. Rowland LM, Summerfelt A, Wijtenburg SA, Du X, Chiappelli JJ, Krishna N, et al. Frontal glutamate and gamma-aminobutyric acid levels and their associations with mismatch negativity and digit sequencing task performance in schizophrenia. JAMA Psychiatry (2016) 73(2):166-74. doi:10.1001/jamapsychiatry.2015.2680

Conflict of Interest Statement: The authors declare that the research was conducted in the absence of any commercial or financial relationships that could be construed as a potential conflict of interest.

Copyright (C) 2017 Marsman, Mandl, Klomp, Cahn, Kahn, Luijten and Hulshoff Pol. This is an open-access article distributed under the terms of the Creative Commons Attribution License (CC BY). The use, distribution or reproduction in other forums is permitted, provided the original author(s) or licensor are credited and that the original publication in this journal is cited, in accordance with accepted academic practice. No use, distribution or reproduction is permitted which does not comply with these terms. 